\title{
Omalizumab in symptomatic therapy of laryngeal oedema and urticaria attacts in a patient with post operative pulmonary carsinoid tumor
}

\author{
Arzu Didem Yalcin*, Kaya Suer \\ From 3rd WAO International Scientific Conference (WISC) 2014 \\ Rio de Janeiro, Brazil. 6-9 December 2014
}

Omalizumab has been investigated in various other conditions including chronic urticaria $(\mathrm{CU})$, perennial and seasonal allergic rhinitis (AR), pruritic bullous pemphigoid, latex allergy, peanut allergy, idiopathic anaphylaxis, hyper-IgE syndrome, chronic rhinosinusitis, interstitial cystitis, aspirin sensitivity, mastocytosis, eosinophilic gastroenteritis and atopic dermatitis. Most patients with chronic urticaria have an autoimmune cause: some patients produce IgE autoantibodies against autoantigens, such as thyroperoxidase or doublestranded DNA, whereas other patients make IgG autoantibodies against FceRI, IgE, or both, which might chronically activate mast cells and basophils.I had a male patients with food allergy with pulmonary carsinoid tumor, aged 23. Autologous Serum Skin Test, Anti Nuclear Antibody,and hepatitis markers (HBsAg, HBsAb, anti HCV HIV, thyroid antibodies were negative in patients. Liver, thyroid, and renal function tests, serum IgG, IgA, IgM, levels were within normal ranges. Skin prick tests (SPTs) were highly positive for kiwi, tometo, fish, orange The specific IgE levels were correlated with the SPTs. Total IgE level were $960 \mathrm{IU} / \mathrm{L}$ (normal range:0-100 IU/L).A mass was defined on lober lobe of left lung on computerized tomography. PET CT SUVmax:6. The patient was operated. In postoperative period, he had recurrent laryngeal oedema and urticaria attacks. Omalizumab treatment planned because of the patient was resistant to antihistaminics and steroids.

For the very first time, we used omalizumab in symptomatic therapy of recurrent laryngeal oedema and urticaria attacts in a patient with post operative pulmonary carsinoid tumor for eight months. During the four years of follow-up, no reccurrens was noted in carsinoid

Near East University, Cyprus (http://creativecommons.org/publicdomain/zero/1.0/) applies to the data made available in this article, unless otherwise stated. 